OPEN

SUBJECT AREAS:

APPLIED PHYSICS

ELECTRONIC DEVICES

QUANTUM DOTS

PHOTONIC DEVICES

Received

28 March 2013

Accepted

2 September 2013

Published

26 September 2013

Correspondence and requests for materials should be addressed to

T.T. (tayagaki@scl. kyoto-u.ac.jp)

\section{Investigation of the open-circuit voltage in solar cells doped with quantum dots}

\author{
Takeshi Tayagaki $^{1,2}$, Yusuke Hoshi $^{3}$ \& Noritaka Usami ${ }^{3}$ \\ ${ }^{1}$ Institute for Chemical Research, Kyoto University, Uji, Kyoto 61 1-001 1, Japan, 2PRESTO-JST, Kawaguchi, Saitama 332-0012, \\ Japan, ${ }^{3}$ Graduate School of Engineering, Nagoya University, Nagoya 464-8603, Japan.
}

Quantum dots (QDs) have attracted much attention for use in photovoltaic applications because of their potential for overcoming the limits of conventional single-junction devices. One problem associated with solar cells using QDs is that the open-circuit voltage $\left(V_{o c}\right)$ always decreases with the addition of QDs with respect to the reference cell without QDs. Here, we report the investigation of current-voltage characteristics in $\mathrm{Ge} / \mathrm{Si} \mathrm{QD}$ solar cells in the temperature range from 100 to $300 \mathrm{~K}$. We show that even though $V_{o c}$ decreases with increasing temperature, it depends on the nominal Ge thickness, indicating that $V_{o c}$ reduction is primarily caused by a decrease in the bandgap energy of the cell. From photoluminescence decay measurements, we found that rapid carrier extraction from QDs occurred in the solar cells; this process eliminates the quasi-Fermi energy splitting between the QDs and the host semiconductor and causes $V_{o c}$ reduction in QD solar cells.

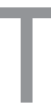
o overcome the limits of conventional single-junction devices ${ }^{1}$, solar cells using quantum dots (QDs) have recently been proposed and extensively studied ${ }^{2-7}$. One promising approach uses the concept of the intermediate-band solar cell ${ }^{8}$, in which sub-bandgap-energy photons are absorbed through transition from the valence band (VB) to the intermediate band (IB) and from the IB to the conduction band (CB) [Fig. 1a]. The generated current augments to the current of conventional photons absorbed through the VB-CB transition. The intermediate-band solar cells can supply a high open-circuit voltage $\left(V_{o c}\right)$, which is determined by the quasiFermi level (QFL) difference between the CB and VB of the host: $q V_{\mathrm{oc}}=E_{F C}-E_{F V}$. Thus, splitting the Fermi levels into three separate quasi-Fermi levels is a critical issue for preserving the high output voltage of the cell ${ }^{9}$.

The energy levels of the confined states in a QD have been proposed for use as an IB ${ }^{4}$. Many QD IB solar cells, which enable the optical absorption of photons with energies below the bandgap of the host, have been reported in attempts to demonstrate highly efficient solar cell devices ${ }^{4,7-17}$. However, to date, all the reported experimental efficiencies of QD solar cells have been less than those of the best single-junction devices. The primary issue in all reports is that $V_{o c}$ decreases with respect to reference cells without QDs, even though the short-circuit current density shows a small increase in devices with QDs. This difference may be caused by the optical quality of the $\mathrm{QD} /$ bulk interface ${ }^{10,12,14}$. In contrast, very recent work on $V_{o c}$ reduction has shown that suppression of the thermal escape that is present at low temperatures results in the recovery of $V_{o c}$ in QD solar cells ${ }^{7,17}$. In previously reported QD solar cells, thermal excitation at room temperature can easily excite the carrier out of the QDs because of the small confinement energy of the $\mathrm{QDs}{ }^{15}$. Such thermal carrier extractions would eliminate the quasi-Fermi energy separation between the IB of QDs and the VB (CB) of the host semiconductors ${ }^{9}$. Thus, optimising the carrier extraction from QDs involves quasi-Fermi energy separation in IB solar cells. This latter process has not been well understood; it remains one of the most critical problems in QD solar cells.

In conventional single-junction solar cells, $V_{o c}$ is, in general, determined using the saturation current, which is denoted as $J_{0}$,

$$
V_{o c}=\frac{m k_{B} T}{q} \ln \left(\frac{J_{s c}}{J_{0}}+1\right),
$$

where $m$ and $J_{s c}$ are the ideality factor and short-circuit current density, respectively ${ }^{1,18} \cdot V_{o c}$ reduction may be related to the enhanced generation of the saturation current, which is determined by various mechanisms, such as the recombination current or diffusion current. In either case, $V_{o c}$ decreases with increasing temperature, as determined by the bandgap energy of the host semiconductor, which is denoted by $E_{g}$, and the dark current characteristics $^{1,18}$

$$
V_{o c}(T)=E_{g} / q-C T
$$




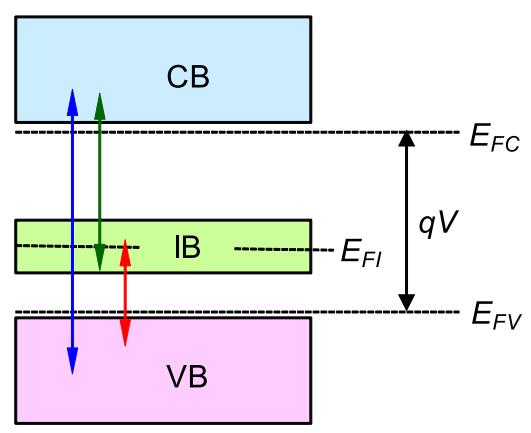

C

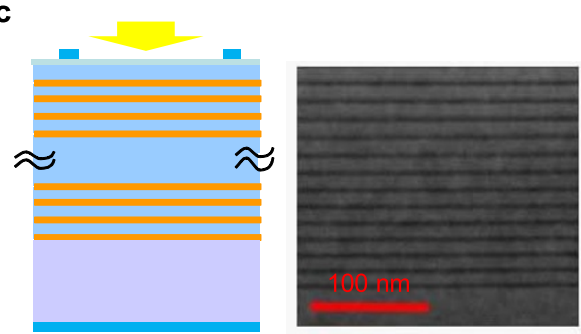

e

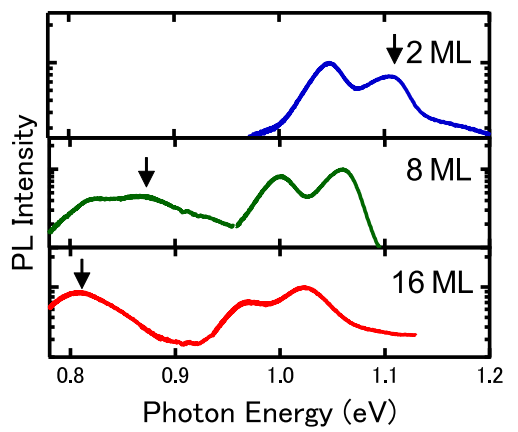

b

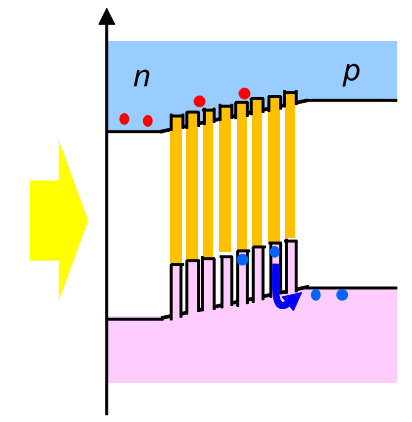

d

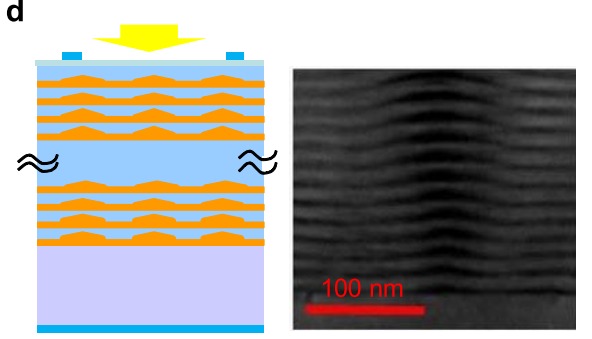

f

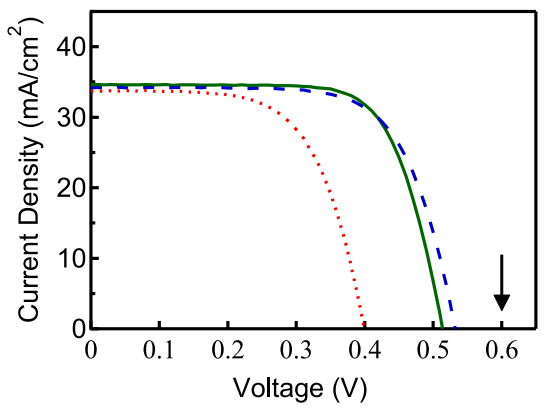

Figure $1 \mid$ Photovoltaic characteristics of Ge/Si solar cells. (a), Band diagram of a solar cell with an intermediate band. $E_{F C}, E_{F V}$, and $E_{F I}$ are the quasiFermi levels for conduction band (CB), valence band (VB), and intermediate band (IB), respectively. (b), Schematic energy diagram of Ge/Si QD solar cells. (c), Schematic of a $c$-Si solar cell with Ge/Si QWs of a nominal Ge thickness of $2 \mathrm{ML}$ and (d), QDs of a nominal Ge thicknesses of 8 ML, with transmission electron microscope images of QWs and QDs. (e), PL spectra of Ge/Si solar cells with different Ge thicknesses at 10 K. Arrows indicate nophonon optical transitions in QWs and QDs. (f), Current-voltage characteristics under illumination at room temperature for nominal Ge thicknesses of 2 MLs (dashed line), 8 MLs (solid line), and 16 MLs (dotted line). The arrow indicates the $V_{o c}$ in a c-Si solar cell.

where $C$ is the temperature coefficient, which reflects the dark current characteristics in the solar cells. In previously reported QD solar cells, $V_{o c}$ reduction is believed to be due to an increase in recombination that is caused by the quality of QDs ${ }^{10,12,14}$, corresponding to an increased temperature coefficient, $C$. However, the detailed $V_{o c}$ mechanism is not well understood.

In this work, we report the investigation of the impact of $\mathrm{Ge} / \mathrm{Si}$ heterostructures on the $V_{o c}$ in Ge/Si QD solar cells and of carrier extraction from QDs. We found that in Ge/Si QD solar cells, $V_{o c}$ at the zero-temperature limit, rather than the temperature coefficient, depends strongly on the nominal Ge thickness of Ge/Si heterostructures. This indicates that the reduced $V_{o c}$ in QD solar cells is not caused simply by enhanced carrier recombination due to the addition of QDs, but is rather caused by the lowering of the bandgap energy itself. Moreover, we found that carrier extraction from QDs is accelerated in the solar cells with respect to that in the as-grown $\mathrm{Ge} / \mathrm{Si}$ QDs because of the inherent electric field, indicating that the rapid carrier extraction eliminates the Fermi energy separation between the QD and the host semiconductor and causes $V_{o c}$ reduction in QD solar cells.

\section{Results}

To study the reduced open-circuit voltage, we used Ge dots in the intrinsic region of Si-based solar cells [Fig. 1b] ${ }^{19-22}$. Ge/Si heterostructures provide a unique opportunity to investigate the optical and electronic properties in artificially controlled nanostructures ${ }^{23-28}$, whereby we can systematically compare these nanostructures with the widely used crystalline $\mathrm{Si}(c-\mathrm{Si})$ solar cells. In addition, $\mathrm{Ge} / \mathrm{Si}$ heterostructures form a type-II band offset, where electrons and holes are spatially separated by heterostructures, which reduces their radiative and nonradiative recombination. The $\mathrm{Ge} / \mathrm{Si}$ heterostructure forms various morphologies, such as quantum wells (QWs) and island-like QDs, depending on the nominal Ge thickness ${ }^{23,24}$. A nominal Ge thickness below $\sim 4$ monolayers (MLs) results in the formation of QWs [Fig. 1c]. In contrast, at thicknesses above 4 MLs, the lattice mismatch between $\mathrm{Si}$ and Ge promotes the formation of island-like QDs [Fig. 1d], a process known as Stranski-Krastanov growth $^{23}$. Moreover, the optical properties of the island-like structures, which result in a strongly confined electronic system, are determined by their structures and differ from those in bulk crystals $^{23,24,29}$. Figure 1e shows the PL spectra of Ge/Si solar cells with 
different nominal Ge thicknesses, measured at $\sim 10 \mathrm{~K}$. In 2-ML Ge layers, corresponding to Ge/Si QWs, the PL peaks, which appear at around $1.1 \mathrm{eV}$, are assigned to transverse-optical-phonon-assisted and no-phonon transitions in the QWs. In contrast, in Ge/Si QDs formed from nominal Ge thicknesses of 8 and 16 MLs, a broad PL band appears at around $0.8 \mathrm{eV}$, which reflects the formation of island-like Ge/Si QDs. From the PL peak position of the no-phonon transition, we can estimate bandgap energies of $\sim 1.11, \sim 0.88$, and $\sim 0.82 \mathrm{eV}$ for 2-ML QWs and 8- and 16-ML QDs, respectively.

Figure 1f shows the current-voltage characteristics under 1-sun illumination in Ge/Si QD and QW solar cells with nominal Ge thicknesses of 2, 8, and $16 \mathrm{ML}$. For comparison, the typical $V_{o c}$ in c-Si cells without $\mathrm{Ge} / \mathrm{Si}$ heterostructures is indicated by the arrow in Fig. 1f. With respect to $c$-Si reference cells, the $V_{o c}$ in $\mathrm{Ge} / \mathrm{Si}$ cells decreases to less than $0.6 \mathrm{eV}$. Although 16-ML solar cells show a significant $V_{o c}$ reduction, the difference in $V_{o c}$ between the 2-ML and 8-ML solar cells is negligible. These results imply that the $V_{o c}$ of the QD solar cells at room temperature is determined both by the bandgap energy of the system, $E_{g}$, and by the temperature coefficient, $C$, as described by equation (2).

To clarify the origin of the $V_{o c}$ reduction in QD solar cells with respect to the reference $c$-Si solar cell, we studied the current-voltage characteristics at various temperatures. The $V_{o c}$ of the QD solar cells was determined from current-voltage measurements under light irradiation. Figure 2a shows the temperature dependence of $V_{o c}$ in $c$-Si solar cells and $\mathrm{Ge} / \mathrm{Si}$ solar cells with different nominal Ge thicknesses. We found that the $V_{o c}$ at low temperatures depends on the $\mathrm{Ge} / \mathrm{Si}$ heterostructure, indicating that $V_{o c}$ reduction originates from the reduced bandgap energy of the system due to the insertion of $\mathrm{Ge} /$ $\mathrm{Si}$ heterostructures. In addition, in all the samples, $V_{o c}$ decreased almost linearly with increasing temperature. Similar to conventional single-junction devices, the dark current decreased at low temperatures in QD solar cells ${ }^{30}$. Thus, these findings indicate that $V_{o c}$ reduction at room temperature originates from both the reduced bandgap energy and the enhanced dark current due to the finite temperature in the cells.

In Fig. 2a, we can see that $V_{o c}$ is well described by equation (2), in accordance with the conventional Shockley-Queisser model ${ }^{1}$. Therefore, to clarify the origin of $V_{o c}$ reduction at room temperature, we fitted the temperature dependence of the open-circuit voltage with the equation $V_{o c}(T)=V_{0}-C T$ and extracted the $V_{0}$ and $C$ parameters for each $\mathrm{Ge} / \mathrm{Si}$ solar cell.

Figure $2 \mathrm{~b}$ illustrates the dependence of the obtained $V_{0}$ and $C$ values on the nominal Ge thickness. The $V_{o c}$ at low temperatures, $V_{0}$, decreased with increasing numbers of Ge layers. This means that the bandgap energy of Ge/Si QD solar cells is reduced with decreasing bandgap energy of Ge/Si QWs and QDs. In contrast, the temperature coefficient $C$ in cells with QDs does not show a significant increase with respect to the reference $c$-Si cell, indicating that the carrier recombination is not significantly enhanced even in solar cells with $\mathrm{Ge} / \mathrm{Si}$ QDs. In addition, a small reduction of the $C$ values occurs with increasing Ge thickness, meaning that $\mathrm{Ge} / \mathrm{Si}$ heterostructures may slightly improve the dark current characteristics because the $C$ reflects the saturation current, $J_{0}$. This may be caused by the type-II band offset in Ge/Si heterostructures where electrons and holes are spatially separated by the heterostructures to reduce carrier recombination. The small variation in $C$ implies that the $V_{o c}$ reduction at room temperature does not match the bandgap changes with the insertion of $\mathrm{Ge} / \mathrm{Si}$ heterostructures, as seen in Fig. 1f. Therefore, in Ge/Si QD solar cells, $V_{o c}$ reduction is primarily caused by the reduced bandgap energy of the system, rather than by enhanced carrier recombination, due to the addition of QDs. The modulation of the temperature coefficient may play a secondary role in $V_{o c}$ reduction, but it offers a promising approach for increasing the $V_{o c}$ at room temperature, as will be discussed later.
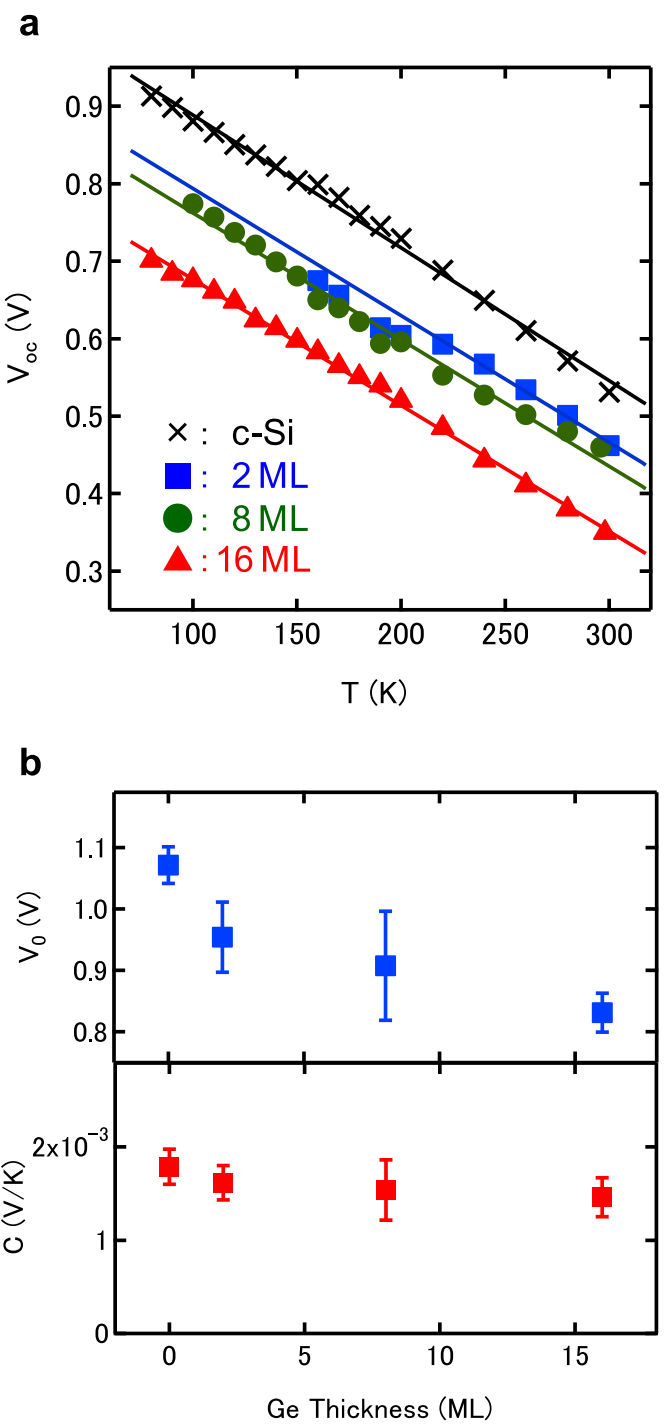

Figure $2 \mid$ Temperature dependence of open-circuit voltage in Ge/Si solar cells. (a), Temperature dependence of $V_{o c}$ in c-Si and Ge/Si solar cells. (b), $V_{o c}$ at the zero-temperature limit, $V_{0}$, and the temperature coefficient, $C$, plotted against the nominal Ge thickness.

Next, to gain further understanding of $V_{o c}$ reduction with the insertion of QDs, we studied carrier extraction from Ge QDs using PL decay measurements. Figure 3a shows the PL decay profiles for both the as-grown $\mathrm{Ge} / \mathrm{Si}$ heterostructures and those used in Si solar cells. Whereas the PL decay times for the as-grown Ge/Si heterostructures are longer than 100 ns, those in the Si solar cells are a few tens of nanoseconds. This indicates that the carriers generated in QDs can be extracted before they are lost by radiative or nonradiative recombination in QDs. Thus, the quality of the QD/bulk interface is not a major source of efficiency loss and has a small effect on $V_{o c}$ reduction in Ge/Si QD solar cells. In addition, as shown in Fig. 3b and the inset, the PL decay profiles of the Ge/Si solar cells depend strongly on the applied electric field. Although the PL decay time is increased with increasing forward-bias voltage, shorter PL decay times are almost unchanged under reverse bias conditions, indicating that carrier extractions are controlled by the inherent electric field applied to the Si $p-i-n$ junctions. These findings indicate the occurrence of rapid carrier extraction from the QD due to the inherent bias field. Such a carrier transfer prevents the occurrence of non-thermal carrier distribution between the QD states and the VB (CB) in the host semiconductors and eliminates the quasi-Fermi energy separation 
a

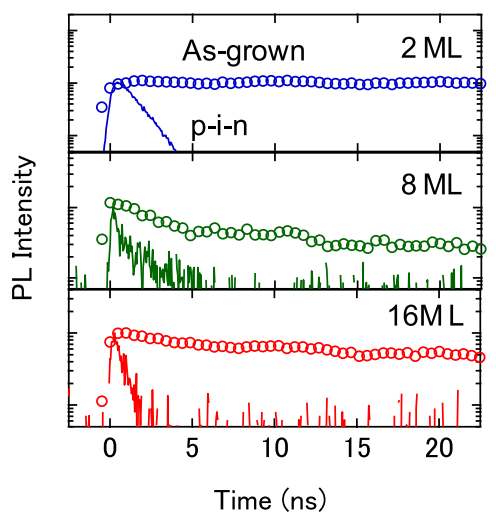

b

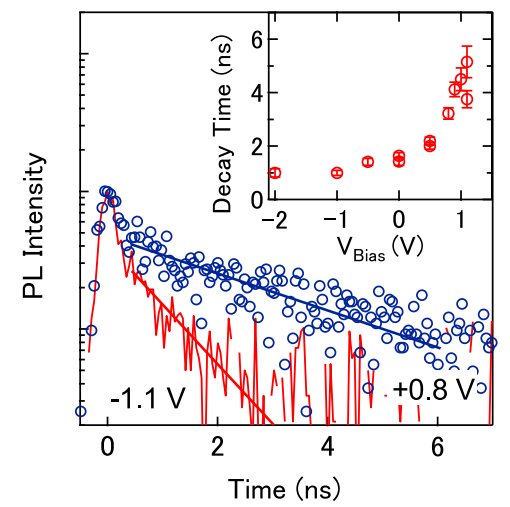

Figure $3 \mid$ Photoluminescence decay profiles in Ge/Si solar cells. (a), PL decay profiles in Ge/Si solar cells (solid curves) and in as-grown Ge/Si QDs (circles) at $10 \mathrm{~K}$. (b), PL decay profiles under forward $(+0.8 \mathrm{~V})$ and reverse $(-1.1 \mathrm{~V})$ bias conditions. Inset: Biased electric field dependence of PL decay time.

between the QDs and the host semiconductor ${ }^{9}$. In IB solar cells, separate quasi-Fermi levels are critical for preserving $V_{o c}$. Therefore, we concluded that in the Ge/Si QD solar cells, $V_{o c}$ reduction is not caused by the quality of QDs but by carrier extraction mechanisms.

\section{Discussion}

We now discuss approaches that can be used for obtaining highly efficient QD solar cells. Our results show that $V_{o c}$ reduction in QD solar cells is primarily caused by a reduced bandgap energy. This corresponds to the fact that the reduced bandgap energy enhances the saturation current in the conventional diode model. As an example, when the saturation current is governed by the recombination current, $J_{0}$ and $V_{o c}$ take the form ${ }^{18}$,

$$
\begin{gathered}
J_{0}=\frac{q W N_{c}^{1 / 2} N_{v}^{1 / 2}}{\sqrt{\tau_{p} \tau_{n}}} \exp \left[-\frac{E_{g}}{k_{B} T}\right] \\
V_{o c} \approx \frac{E_{g}}{q}-\frac{2 k_{B} T}{q} \ln \left[\frac{q W N_{c}^{1 / 2} N_{v}^{1 / 2}}{J_{s c} \sqrt{\tau_{p} \tau_{n}}}\right],
\end{gathered}
$$

where $W, N_{c(v)}$, and $\tau_{p(n)}$ are the total depletion width, effective conduction (valence) band density of states, and lifetime of minority holes (electrons), respectively. Therefore, to compensate for the loss due to the reduced bandgap energy, the saturation current has to be reduced. First, from equation (3), we expect that the modification of carrier lifetimes using $\mathrm{Ge} / \mathrm{Si}$ heterostructures can reduce the saturation current, which also results in a smaller temperature coefficient,
$C$, as indicated by equation (4). Such an enhanced carrier lifetime is expected in type II quantum confinement due to electron-hole separation, as seen in Fig. 2b. Secondly, the effective densities of states, $N_{c}$ and $N_{v}$, can also be reduced by the quantum confinement effect, forming a discrete electronic structure. Thus, reduction of $C$ in designed heterostructures could be a promising approach for preserving the $V_{o c}$ at room temperature.

To realise the concept of an IB solar cell, such a discrete electronic structure under quantum confinement might also be necessary because, in the intermediate band solar cell, the quasi-Fermi energy splitting between the IB and the VB (CB) is required to maintain the $V_{o c}$ in the host semiconductor ${ }^{9}$. Such discrete energy levels in smaller QDs may break the thermal connection between the QD states and the VB (CB) in the host semiconductor, enabling the quasi-Fermi energy splitting. Furthermore, instead of the thermal carrier extraction, which causes $V_{o c}$ reduction via reduced Fermi energy separation between the QDs and the host semiconductor, carrier extractions using two-step two-photon absorption ${ }^{8,15}$ and Auger recombination $^{22}$ should be enhanced to demonstrate the IB operation.

In the present work, we demonstrate that $V_{o c}$ reduction in $\mathrm{Ge} / \mathrm{Si}$ QD solar cells is caused by the reduced bandgap energy of the QD solar cell, rather than by enhanced carrier recombination due to the insertion of QDs. The temperature coefficient remains almost unchanged, even in QD solar cells, and probably functions as the critical parameter for maintaining the value of $V_{o c}$. Our findings indicate that $V_{o c}$ reduction can be mitigated by tuning the temperature coefficient, which reflects the saturation current of the $p-i-n$ junction. Controlling the saturation current is critical for producing highly efficient nanostructure in solar cells. One promising approach for realising this control is to tune the electronic properties, such as the density of states, carrier lifetime, or carrier extraction dynamics, in designed nanostructures.

\section{Methods}

Samples were grown using a gas-source molecular beam epitaxy system (Air-Water VCE S2020) with disilane and germane as the gas sources on $p$-type $\mathrm{Si}(100)$ substrates with resistivities of $0.8-1.2 \Omega \mathrm{cm}^{23}$. Ge/Si layers stacked in a 50-layer structure were separated by $20-\mathrm{nm}$-thick Si spacer layers. Ge/Si QD solar cells were formed using the rapid thermal annealing process described in our previous paper $^{21}$. The photocurrent-voltage behaviours at room temperature were measured using a solar cell simulator. At low temperatures, we used a Xe lamp with filters (Oriel, Air Mass Filter, AM 1.5 Global) at a $\sim 1$-sun intensity as the light source. The photocurrent was measured directly with a computer-interfaced Keithley 2636A source measurement unit. PL measurements were conducted at $\sim 10 \mathrm{~K}$ under zero-bias conditions. The second harmonic of a 2-MHz cavity-dumped Ti:sapphire laser $(3.1 \mathrm{eV})$ was used as the excitation light source ${ }^{31}$. Time-integrated PL spectra were acquired using an InGaAs array detector. The PL decay time was measured using a photomultiplierequipped photon-counting system. In the 8-and 16-ML samples, the temporal changes of the PL intensities of island-like QDs at $\sim 0.8 \mathrm{eV}$ were measured. In the 2ML sample, the PL intensity of the QWs at $\sim 1 \mathrm{eV}$ was used.

1. Shockley, W. \& Queisser, H. J. Detailed balance limit of efficiency of p-n junction solar cells. J. Appl. Phys. 32, 510-519 (1960).

2. Aroutiounian, V., Petrosyan, S., Khachatryan, A. \& Touryan, K. Quantum dot solar cells. J. Appl. Phys. 89, 2268-2271 (2001).

3. Nozik, A. J. Quantum dot solar cells. Physica E 14, 115-120 (2002).

4. Martí, A. et al. Production of photocurrent due to intermediate-to-conductionband transitions: a demonstration of a key operating principle of the intermediate-band solar cell. Phys. Rev. Lett. 97, 247701 (2006).

5. Wang, X. et al. Tandem colloidal quantum dot solar cells employing a graded recombination layer. Nat. Photon. 5, 480-484 (2011).

6. Semonin, O. E. et al. Peak external photocurrent quantum efficiency exceeding $100 \%$ via MEG in a quantum dot solar cell. Science 334, 1530-1533 (2011).

7. Luque, A., Martí, A. \& Stanley, C. Photovoltaics: Towards the intermediate band. Nat. Photon. 6, 146 (2012).

8. Luque, A. \& Martí, A. Increasing the efficiency of ideal solar cells by photon induced transitions at intermediate levels. Phys. Rev. Lett. 78, 5014-5017 (1997).

9. Luque, A. et al. Experimental analysis of the quasi-Fermi level splits in quantum dot intermediate-band solar cells. Appl. Phys. Lett. 87, 083505 (2005).

10. Hubbard, S. M. et al. Effect of strain compensation on quantum dot enhanced GaAs solar cells. Appl. Phys. Lett. 92, 123512 (2008).

11. Oshima, R., Takata, A. \& Okada, Y. Strain-compensated InAs/GaNAs quantum dots for use in high-efficiency solar cells. Appl. Phys. Lett. 93, 083111 (2008). 
12. Popescu, V., Bester, G., Hanna, M. C., Norman, A. G. \& Zunger, A. Theoretical and experimental examination of the intermediate-band concept for strainbalanced (In,Ga)As/Ga(As,P) quantum dot solar cells. Phys. Rev. B 78, 205321 (2008).

13. Blokhin, S. A. et al. AlGaAs/GaAs photovoltaic cells with an array of InGaAs QDs. Semiconductors 43, 514-518 (2009).

14. Guimard, D. et al. Y. Fabrication of InAs/GaAs quantum dot solar cells with enhanced photocurrent and without degradation of open circuit voltage. Appl. Phys. Lett. 96, 203507 (2010).

15. Okada, Y. et al. Increase in photocurrent by optical transitions via intermediate quantum states in direct-doped InAs/GaNAs strain-compensated quantum dot solar cell. J. Appl. Phys. 109, 024301 (2011)

16. Bailey, C. G., Forbes, D. V., Raffaelle, R. P. \& Hubbard, S. M. Near 1 V open circuit voltage InAs/GaAs quantum dot solar cells. Appl. Phys. Lett. 98, 163105 (2011).

17. Linares, P. G. et al. Voltage recovery in intermediate band solar cells. Sol. Energ. Mater. Sol. C. 98, 240-244 (2012).

18. Sze, S. M. Physics of Semiconductor Devices (John Wily \& Suns, 1981).

19. Alguno, A. et al. Enhanced quantum efficiency of solar cells with self-assembled Ge dots stacked in multilayer structure. Appl. Phys. Lett. 83, 1258-1260 (2003).

20. Kechiantz, A. M., Kocharyan, L. M. \& Kechiyants, H. M. Band alignment and conversion efficiency in $\mathrm{Si} / \mathrm{Ge}$ type-II quantum dot intermediate band solar cells. Nanotechnology 18, 405401 (2007).

21. Usami, N. et al. Simultaneous enhanced photon capture and carrier generation in Si solar cells using Ge quantum dot photonic nanocrystals. Nanotechnology 23, 185401 (2012)

22. Tayagaki, T. et al. Enhanced carrier extraction from Ge quantum dots in Si solar cells under strong photoexcitation. Appl. Phys. Lett. 101, 133905 (2012).

23. Sunamura, H., Usami, N., Shiraki, Y. \& Fukatsu, S. Island formation during growth of $\mathrm{Ge}$ on $\mathrm{Si}(100)$ : a study using photoluminescence spectroscopy. Appl. Phys. Lett. 66, 3024 (1995).

24. Brunner, K. Si/Ge nanostructures. Rep. Prog. Phys. 65, 27 (2002).

25. Zhang, J. J. et al. SiGe growth on patterned Si(001) substrates: Surface evolution and evidence of modified island coarsening. Appl. Phys. Lett. 91, 173115 (2007).

26. Miyamoto, S. et al. Excitonic Aharonov-Bohm effect in isotopically pure ${ }^{70} \mathrm{Ge} / \mathrm{Si}$ self-assembled type-II quantum dots. Phys. Rev. B 82, 073306 (2010).

27. Tayagaki, T., Fukatsu, S. \& Kanemitsu, Y. Photoluminescence dynamics and reduced Auger recombination in $\mathrm{Si}_{1-x} \mathrm{Ge}_{x} / \mathrm{Si}$ superlattices under high-density photoexcitation. Phys. Rev. B 79, 041301(R) (2009).
28. Zhang, J. J. et al. Monolithic growth of ultrathin Ge nanowires on $\mathrm{Si}(001)$. Phys Rev. Lett. 109, 085502 (2012).

29. Yoffe, A. D. Semiconductor quantum dots and related systems: electronic, optical, luminescence and related properties of low dimensional systems. Adv. Phys. 50, 1 (2001).

30. Lu, H. F. et al. Temperature dependence of dark current properties of InGaAs/ GaAs quantum dot solar cells. Appl. Phys. Lett. 98, 183509 (2011).

31. Tayagaki, T., Ueda, K., Fukatsu, S. \& Kanemitsu, Y. Recombination dynamics of high-density photocarriers in type-II Ge/Si quantum dots. J. Phys. Soc. Jpn 81, $064712(2012)$

\section{Acknowledgements}

The authors thank W. Pan for sharing expertise on the preparation of solar cells and T. Kiguchi for measuring transmission electron microscope images. This work was supported by the JST ALCA program. T.T. was supported by the JST PRESTO program.

\section{Author contributions}

T.T. and N.U. conceived the project. Y.H. fabricated the samples. T.T. and Y.H. performed the experiments. T.T. performed data analysis, and wrote the manuscript. All authors discussed the results and commented on the manuscript.

\section{Additional information}

Competing financial interests: The authors declare no competing financial interests.

How to cite this article: Tayagaki, T., Hoshi, Y. \& Usami, N. Investigation of the open-circuit voltage in solar cells doped with quantum dots. Sci. Rep. 3, 2703; DOI:10.1038/ srep02703 (2013).

(c) (i) () $\odot$ This work is licensed under a Creative Commons Attribution-

NonCommercial-NoDerivs 3.0 Unported license. To view a copy of this license, visit http://creativecommons.org/licenses/by-nc-nd/3.0 